\title{
Statistical convergence in probabilistic generalized metric spaces w.r.t. strong topology
}

\section{Rasoul Abazari ${ }^{1 *}$}

"Correspondence:

r.abazari@iauardabil.ac.ir; rasoulabazari1361@gmail.com

'Department of Mathematics, Ardabil Branch, Islamic Azad

University, Ardabil, Iran

\section{Springer}

\begin{abstract}
In this paper, the concept of probabilistic $g$-metric space with degree I, which is a generalization of probabilistic G-metric space, is introduced. Then, by endowing strong topology, the definition of /-dimensional asymptotic density of a subset $A$ of $\mathbb{N}^{\prime}$ is used to introduce a statistically convergent and Cauchy sequence and to study some basic facts.
\end{abstract}

MSC: 40A35; 54E70; 54E35

Keywords: Probabilistic metric space; Generalized metric space; Statistical convergence; Statistical Cauchy sequence; Strong topology

\section{Introduction}

The theory of probabilistic metric space ( $P M$-space) as a generalization of ordinary metric space was introduced by Menger in [12]. In this space, distribution functions are considered as the distance of a pair of points in statistics rather than deterministic.

The concept of the generalized metric space (briefly G-metric space) was introduced by Mustafa and Sims in 2006 [16]. Then, in 2014, Zhou et al. [26] generalized the notion of $P M$-space to the $G$-metric spaces and defined the probabilistic generalized metric space which is denoted by $P G M$-space.

In [3], Choi et al. proposed a generalization of $G$-metric space named $g$-metric space with degree $l$, in which the distance function with degrees $l=1,2$ is equivalent to ordinary and $G$-metric, respectively.

The idea of statistical convergence was first introduced by Steinhaus [25] for real sequences and developed by Fast [7], then reintroduced by Shoenberg [22]. Many authors, such as $[4,6,8,9,17,21]$, have discussed and developed this concept. The theory of statistical convergence has many applications in various fields such as approximation theory [5], finitely additive set functions [4], trigonometric series [27], and locally convex spaces [11].

In 2008, Sencimen and Pehlivan [24] introduced the concepts of statistically convergent sequence and statistically Cauchy sequence in the probabilistic metric space endowed with strong topology.

(c) The Author(s) 2021. This article is licensed under a Creative Commons Attribution 4.0 International License, which permits use, sharing, adaptation, distribution and reproduction in any medium or format, as long as you give appropriate credit to the original author(s) and the source, provide a link to the Creative Commons licence, and indicate if changes were made. The images or other third party material in this article are included in the article's Creative Commons licence, unless indicated otherwise in a credit line to the material. If material is not included in the article's Creative Commons licence and your intended use is not permitted by statutory regulation or exceeds the permitted use, you will need to obtain permission directly from the copyright holder. To view a copy of this licence, visit http://creativecommons.org/licenses/by/4.0/ 
The purpose of this paper is to develop a concept to generalize the probabilistic G-metric space to the probabilistic $g$-metric space with degree $l$. Here, the notation of the generalized space is still referred as $P G M$-space. The $l$-dimensional asymptotic density of a subset $A$ of $\mathbb{N}^{l}$ defined previously by the author in [1] is used to introduce the statistically convergent and Cauchy sequences with respect to strong topology, and some basic facts are studied. Note that in this definition $l=1$ and $l=2$ values coincide exactly with the statistical convergence in $P M$-space and $P G M$-space (related to G-metric), respectively. Thus, the definitions and the obtained results show that this study is more comprehensive.

\section{Preliminaries}

In this section, some basic definitions and results related to $P M$-space, $P G M$-space, and statistical convergence are presented and discussed. First, recall the definition of triangular norm $(t$-norm) as follows.

Definition 2.1 ([23]) A mapping $T:[0,1] \times[0,1] \rightarrow[0,1]$ is called a continuous $t$-norm if $T$ satisfies the following conditions:

(i) $T$ is commutative and associative, i.e., $T(a, b)=T(b, a)$ and $T(a, T(b, c))=T(T(a, b), c)$ for all $a, b, c \in[0,1] ;$

(ii) $T$ is continuous;

(iii) $T(a, 1)=a$ for all $a \in[0,1]$;

(iv) $T(a, b) \leq T(c, d)$ whenever $a \leq c$ and $b \leq d$ for all $a, b, c, d \in[0,1]$.

A distribution function $F$ is a map from extended reals $\mathbb{R}_{\infty}:=\mathbb{R} \cup\{-\infty, \infty\}$ into $[0,1]$ such that it is nondecreasing, left-continuous at every real number, and $F(-\infty)=0$ and $F(\infty)=1$. The set of all distribution functions is denoted by $\Delta$ and $\Delta^{+}:=\{F \in \Delta: F(0)=0\}$.

Definition 2.2 ([23]) A Menger probabilistic metric space (PM-space) is a triple $(X, F, T)$, where $X$ is a nonempty set, $T$ is a continuous $t$-norm. and $F$ is a mapping from $X \times X \rightarrow \Delta^{+}$ satisfying the following conditions:

$\left(F_{(x, y)}\right.$ denotes the value of $F$ at the pair $\left.(x, y)\right)$

(i) $F_{(x, y)}(t)=1$ for all $x, y \in X$ and $t>0$ if and only if $x=y$;

(ii) $F_{(x, y)}(t)=F_{(y, x)}(t)$;

(iii) $F_{(x, y)}(t+s) \geq T\left(F_{(x, z)}(t), F_{(z, y)}(s)\right)$ for all $x, y, z \in X$ and $t, s \geq 0$.

Definition 2.3 ([16]) Let $X$ be a nonempty set and $G: X \times X \times X \rightarrow \mathbb{R}^{+}$, be a function satisfying:

1) $G(x, y, z)=0$ if $x=y=z$

2) $0<G(x, x, y)$ for all $x, y \in X$ with $x \neq y$;

3) $G(x, x, y) \leq G(x, y, z)$ for all $x, y, z \in X$ with $z \neq y$;

4) $G(x, y, z)=G(x, z, y)=G(y, z, x)=\cdots$ (symmetry in all three variables);

5) $G(x, y, z) \leq G(x, a, a)+G(a, y, z)$ for all $x, y, z, a \in X$.

Then the pair $(X, G)$ is called $G$-metric space.

The following definition is a developing of $P M$-space on G-metric.

Definition 2.4 ([26]) A Menger probabilistic G-metric space (PGM-space) is a triple $\left(X, G^{*}, T\right)$, where $X$ is a nonempty set, $T$ is a continuous $t$-norm, and $G^{*}$ is a mapping from $X \times X \times X$ into $\Delta^{+}$, satisfying the following conditions: 
(i) $G_{(x, y, z)}^{*}(t)=1$ for all $x, y, z \in X$ and $t>0$ if and only if $x=y=z$;

(ii) $G_{(x, x, y)}^{*}(t) \geq G_{(x, y, z)}^{*}(t)$ for all $x, y \in X$ with $z \neq y$ and $t>0$;

(iii) $G_{(x, y, z)}^{*}(t)=G_{(x, z, y)}^{*}(t)=G_{(y, x, z)}^{*}(t)=\cdots$ (symmetry in all three variables);

(iv) $G_{(x, y, z)}^{*}(t+s) \geq T\left(G_{(x, a, a)}^{*}(t), G_{(a, y, z)}^{*}(s)\right)$ for all $x, y, z, a \in X$ and $s, t \geq 0$.

Definition 2.5 ([26]) Let $\left(X, G^{*}, T\right)$ be a $P G M$-space and $x_{0} \in X$. For $\epsilon>0$ and $0<\delta<1$, the $(\epsilon, \delta)$-neighborhood of $x_{0}$ is defined as follows:

$$
N_{x_{0}}(\epsilon, \delta)=\left\{y \in X: G_{\left(x_{0}, y, y\right)}^{*}(\epsilon)>1-\delta, G_{\left(y, x_{0}, x_{0}\right)}^{*}(\epsilon)>1-\delta\right\} .
$$

Definition 2.6 ([26])

(i) A sequence $\left\{x_{n}\right\}$ in a $P G M$-space $\left(X, G^{*}, T\right)$ is said to be convergent to a point $x \in X$ if, for every $\epsilon>0$ and $0<\delta<1$, there exists a positive integer $M_{\epsilon, \delta}$ such that $x_{n} \in N_{x}(\epsilon, \delta)$ whenever $n>M_{\epsilon, \delta}$.

(ii) A sequence $\left\{x_{n}\right\}$ in a $P G M$-space $\left(X, G^{*}, T\right)$ is called a Cauchy sequence if, for every $\epsilon>0$ and $0<\delta<1$, there exists a positive integer $M_{\epsilon, \delta}$ such that $G_{\left(x_{m}, x_{n}, x_{l}\right)}^{*}(\epsilon)>1-\delta$ whenever $m, n, l>M_{\epsilon, \delta}$.

(iii) A PGM-space $\left(X, G^{*}, T\right)$ is said to be complete if every Cauchy sequence in $X$ converges to a point in $X$.

In the following, some basic concepts of statistical convergence are discussed.

Definition 2.7 ([7]) Let $A \subset \mathbb{N}$ and $A(n)=\{k \in A ; k \leq n\}$. Then the asymptotic density of $A$ is defined as follows:

$$
\delta(A)=\lim _{n \rightarrow \infty} \frac{|A(n)|}{n} .
$$

For a subset $A$ of $\mathbb{N}$, if $\delta(A)=1$, then it is said to be statistically dense. It is clear that $\delta(\mathbb{N}-A)=1-\delta(A)$.

Definition 2.8 ([7]) A sequence $\left\{x_{n}\right\}$ in $\mathbb{R}$ is said to be statistically convergent to a point $x$ in $\mathbb{R}$ if, for each $\epsilon>0$,

$$
\lim _{n \rightarrow \infty} \frac{1}{n}\left|\left\{k \leq n:\left|x_{k}-x\right| \geq \epsilon\right\}\right|=0 .
$$

For more information about statistical convergence, the references $[2,4,7-10,13-15$, 18-20] can be addressed.

\section{Main results}

In this section the main definitions and results are introduced and discussed. First of all, consider the following definition which is a generalization of a $G$-metric space to an $l$ dimensional case, where $l \in \mathbb{N}$.

Definition 3.1 ([3]) Let $X$ be a nonempty set. A function $g: X^{l+1} \longrightarrow \mathbb{R}_{+}$is called a $g$ metric with degree $l$ on $X$ if it satisfies the following conditions:

g1) $g\left(x_{0}, x_{1}, \ldots, x_{l}\right)=0$ if and only if $x_{0}=x_{1}=\cdots=x_{l}$,

g2) $g\left(x_{0}, x_{1}, \ldots, x_{l}\right)=g\left(x_{\sigma(0)}, x_{\sigma(1)}, \ldots, \ldots, x_{\sigma(l)}\right)$ for permutation $\sigma$ on $\{0,1, \ldots, l\}$, 
g3) $g\left(x_{0}, x_{1}, \ldots, x_{l}\right) \leq g\left(y_{0}, y_{1}, \ldots, y_{l}\right)$ for all $\left(x_{0}, x_{1}, \ldots, x_{l}\right),\left(y_{0}, y_{1}, \ldots, y_{l}\right) \in X^{l+1}$ with $\left\{x_{i}: i=0,1, \ldots, l\right\} \subseteq\left\{y_{i}: i=0,1, \ldots, l\right\}$,

g4) For all $x_{0}, x_{1}, \ldots, x_{s}, y_{0}, y_{1}, \ldots, y_{t}, w \in X$ with $s+t+1=l$,

$$
g\left(x_{0}, x_{1}, \ldots, x_{s}, y_{0}, y_{1}, \ldots, y_{t}\right) \leq g\left(x_{0}, x_{1}, \ldots, x_{s}, w, w, \ldots, w\right)+g\left(y_{0}, y_{1}, \ldots, y_{t}, w, w, \ldots, w\right)
$$

The pair $(X, g)$ is called a $g$-metric space. It is noteworthy that, if $l=1$ (resp. $l=2)$, then it is equivalent to an ordinary metric space (resp. G-metric space).

Definition $3.2([3])$ Let $(X, g)$ be a $g$-metric space, $x \in X$ be a point, and $\left\{x_{k}\right\} \subseteq X$ be a sequence.

1) $\left\{x_{k}\right\} g$-converges to $x$ if for all $\epsilon>0$ there exists $N \in \mathbb{N}$ such that

$$
i_{1}, \ldots, i_{l} \geq N \Rightarrow g\left(x, x_{1}, \ldots, x_{l}\right)<\epsilon
$$

2) $\left\{x_{k}\right\}$ is said to be $g$-Cauchy if for all $\epsilon>0$ there exists $N \in \mathbb{N}$ such that

$$
i_{0}, i_{1}, \ldots, i_{l} \geq N \quad \Longrightarrow \quad g\left(x_{i_{0}}, x_{i_{1}}, \ldots, x_{i_{l}}\right)<\epsilon
$$

3) $(X, g)$ is complete if every $g$-Cauchy sequence in $(X, g)$ is $g$-convergent in $(X, g)$.

Now, by equipping Definition 2.4 with $g$-metric, we introduce the following definition that is a generalization.

Definition 3.3 A Menger probabilistic $g$-metric space (still is denoted as PGM-space) is a triple $(X, F, T)$, where $X$ is a nonempty set, $T$ is a continuous $t$-norm, and $F$ is a mapping from $X^{l+1}$ into $\Delta^{+}$, satisfying the following conditions:

(i) $F_{\left(x_{0}, x_{1}, \ldots, x_{l}\right)}(t)=1$ for all $x_{0}, x_{1}, \ldots, x_{l} \in X$ and $t>0$ if and only if $x_{0}=x_{1}=\cdots=x_{l}$;

(ii) $F_{\left(x_{0}, x_{1}, \ldots, x_{l}\right)}(t) \geq F_{\left(y_{0}, y_{1}, \ldots, y_{l}\right)}(t)$ for all $\left(x_{0}, x_{1}, \ldots, x_{l}\right),\left(y_{0}, y_{1}, \ldots, y_{l}\right) \in X^{l+1}$ with $\left\{x_{i}: i=0,1, \ldots, l\right\} \subseteq\left\{y_{i}: i=0,1, \ldots, l\right\}$

(iii) $F_{\left(x_{0}, x_{1}, \ldots, x_{l}\right)}(t)=F_{\left(x_{\sigma(0)}, x_{\sigma(1)}, \ldots, \ldots, x_{\sigma(l)}\right)}(t)$ for permutation $\sigma$ on $\{0,1, \ldots, l\}$;

(iv) For all $x_{0}, x_{1}, \ldots, x_{m}, y_{0}, y_{1}, \ldots, y_{n}, w \in X$ with $m+n+1=l$,

$$
F_{\left(x_{0}, x_{1}, \ldots, x_{m}, y_{0}, y_{1}, \ldots, y_{n}\right)}(t+s) \geq T\left(F_{\left(x_{0}, x_{1}, \ldots, x_{m}, w, w, \ldots, w\right)}(t), F_{\left(y_{0}, y_{1}, \ldots, y_{n}, w, w, \ldots, w\right)}(s)\right) .
$$

In the following, according to the generalization of asymptotic density given in [1], statistically convergent and Cauchy sequences in a PGM-space are introduced.

Definition 3.4 Let $(X, F, T)$ be a $P G M$-space. For any $\epsilon>0,0<\delta<1$ and $x \in X$, the strong $(\epsilon, \delta)$-vicinity of $x$ is defined by the subset $M_{x}(\epsilon, \delta)$ of $X^{l}$ as follows:

$$
M_{x}(\epsilon, \delta)=\left\{\left(x_{1}, x_{2}, \ldots, x_{l}\right) \in X^{l} ; F_{\left(x, x_{1}, x_{2}, \ldots, x_{l}\right)}(\epsilon)>1-\delta\right\}
$$

Next, we generalize the concept of asymptotic density of a set in an $l$-dimensional case.

Definition 3.5 Let $K \subset \mathbb{N}^{l}$, the l-dimensional asymptotic density of $K$ is defined by

$$
\delta_{l}(K)=\lim _{n \rightarrow \infty} \frac{l !}{n^{l}}\left|\left\{\left(i_{1}, i_{2}, \ldots, i_{l}\right) \in K ; i_{1}, i_{2}, \ldots, i_{l} \leq n\right\}\right| .
$$


Definition 3.6 Let $(X, F, T)$ be a $P G M$-space.

(i) A sequence $\left\{x_{n}\right\}$ in $X$ is statistically convergent to a point $x$ in $X$ w.r.t. strong topology if, for any $\epsilon>0$ and $0<\delta<1$,

$$
\delta_{l}\left(\left\{\left(i_{1}, i_{2}, \ldots, i_{l}\right) \in \mathbb{N}^{l}: F_{\left(x_{i_{1}}, x_{i_{2}}, \ldots, x_{i}, x\right)}(\epsilon) \leq 1-\delta\right\}\right)=0
$$

and is denoted by $x_{n} \stackrel{s t}{\longrightarrow} x$ or $s t-\lim _{n \rightarrow \infty} x_{n}=x$.

(ii) $\left\{x_{n}\right\}$ is said to be statistically Cauchy w.r.t. strong topology if, for all $\epsilon>0$ and $0<\delta<1$, there exists $i_{\epsilon} \in \mathbb{N}$ such that

$$
\delta_{l}\left(\left\{\left(i_{1}, i_{2}, \ldots, i_{l}\right) \in \mathbb{N}^{l}: F_{\left(x_{i_{1}}, x_{i_{2}}, \ldots, x_{i_{l}}, x_{i_{\epsilon}}\right)}(\epsilon) \leq 1-\delta\right\}\right)=0 .
$$

We can restate part $(i)$ of the above definition as follows:

$\left(i^{\prime}\right) x_{n} \stackrel{s t}{\longrightarrow} x$ if and only if, for any $\epsilon>0$ and $0<\delta<1$,

$$
\delta_{l}\left(\left\{\left(i_{1}, i_{2}, \ldots, i_{l}\right) \in \mathbb{N}^{l}:\left(x_{i_{1}}, x_{i_{2}}, \ldots, x_{i_{l}}\right) \notin M_{x}(\epsilon, \delta)\right\}\right)=0 .
$$

The following theorem shows that if a sequence is statistically convergent to a point in $X$, then that point is unique.

Theorem 3.7 Let $\left\{x_{n}\right\}$ be a sequence in a PGM-space $(X, F, T)$ such that $x_{n} \stackrel{s t}{\longrightarrow} x$ and $x_{n} \stackrel{s t}{\longrightarrow} y$, then $x=y$.

Proof Let $\epsilon>0$ and $0<\delta<1$, by the continuity of $T$, there exists $0<\delta_{0}<1$ such that

$$
T\left(1-\delta_{0}, 1-\delta_{0}\right)>1-\delta
$$

Set

$$
\begin{aligned}
& A(\epsilon, \delta):=\left\{\left(i_{1}, i_{2}, \ldots, i_{l}\right) \in \mathbb{N}^{l}: F_{\left(x_{i_{1}}, x_{i_{2}}, \ldots, x_{i}, x\right)}\left(\frac{\epsilon}{2}\right) \leq 1-\delta_{0}\right\}, \\
& B(\epsilon, \delta):=\left\{\left(i_{1}, i_{2}, \ldots, i_{l}\right) \in \mathbb{N}^{l}: F_{\left(x_{i_{1}}, x_{i_{2}}, \ldots, x_{i}, y\right)}\left(\frac{\epsilon}{2}\right) \leq 1-\delta_{0}\right\},
\end{aligned}
$$

and

$$
C(\epsilon, \delta):=A(\epsilon, \delta) \cup B(\epsilon, \delta) .
$$

Since $x_{n} \stackrel{s t}{\longrightarrow} x$ and $x_{n} \stackrel{s t}{\longrightarrow} y$, so $\delta_{l}(A(\epsilon, \delta))=\delta_{l}(B(\epsilon, \delta))=0$ and hence $\delta_{l}(C(\epsilon, \delta))=0$, therefore $\delta_{l}\left(C^{c}(\epsilon, \delta)\right)=1$. Suppose $\left(i_{1}, i_{2}, \ldots, i_{l}\right) \in C^{c}(\epsilon, \delta)$, then by parts (ii) of Definition 3.3 and (iv) of Definition 2.1 we have

$$
\begin{aligned}
F_{(x, y, y, \ldots, y)}(\epsilon) & \geq T\left(F_{\left(x_{i_{1}}, x_{i_{1}}, \ldots, x_{i_{1}}, x\right)}\left(\frac{\epsilon}{2}\right), F_{\left(x_{i_{1}}, y, y, \ldots, y\right)}\left(\frac{\epsilon}{2}\right)\right) \\
& \geq T\left(F_{\left(x_{i_{1}}, x_{i_{2}}, \ldots, x_{i}, x\right)}\left(\frac{\epsilon}{2}\right), F_{\left(x_{i_{1}}, x_{i_{2}}, \ldots, x_{i}, y\right)}\left(\frac{\epsilon}{2}\right)\right)
\end{aligned}
$$




$$
\begin{aligned}
& >T\left(1-\delta_{0}, 1-\delta_{0}\right) \\
& >1-\delta
\end{aligned}
$$

Since $\delta>0$ is arbitrary, we conclude that $F_{(x, y, y, \ldots, y)}(\epsilon)=1$, and therefore $x=y$.

Theorem 3.8 Every convergent sequence in a PGM-space is statistically convergent.

Proof Let $\left\{x_{n}\right\}$ be a sequence in the $P G M$-space $(X, F, T)$ that converges to a point $x \in X$. For $\epsilon>0$ and $0<\delta<1$, there exists $n_{0} \in \mathbb{N}$ such that, for all $i_{1}, i_{2}, \ldots, i_{l} \geq n_{0}$,

$$
F_{\left(x_{i_{1}}, x_{i_{2}}, \ldots, x_{i}, x\right)}(\epsilon)>1-\delta .
$$

Set

$$
A(n):=\left\{\left(i_{1}, i_{2}, \ldots, i_{l}\right) \in \mathbb{N}^{l}: i_{1}, i_{2}, \ldots, i_{l} \leq n, F_{\left(x_{i_{1}}, x_{i_{2}}, \ldots, x_{i}, x\right)}(\epsilon)>1-\delta\right\},
$$

then

$$
|A(n)| \geq\left(\begin{array}{c}
n-n_{0} \\
l
\end{array}\right)
$$

and

$$
\lim _{n \rightarrow \infty} \frac{l !|A(n)|}{n^{l}} \geq \lim _{n \rightarrow \infty} \frac{l !}{n^{l}}\left(\begin{array}{c}
n-n_{0} \\
l
\end{array}\right)=1
$$

so

$$
s t-\lim _{n \rightarrow \infty} x_{n}=x .
$$

Example 3.9 shows that the converse of the above theorem is not valid.

Example 3.9 Let $X=\mathbb{R}$ and $G: \mathbb{R} \times \mathbb{R} \times \mathbb{R} \longrightarrow \mathbb{R}^{+}$be a $G$-metric on $\mathbb{R}$ defined by

$$
G(x, y, z)=|x-y|+|x-z|+|y-z| .
$$

$(T=\min )$ Define a function $F: \mathbb{R} \times \mathbb{R} \times \mathbb{R} \longrightarrow \mathbb{R}^{+}$as follows:

$$
F_{(x, y, z)}(t)= \begin{cases}H(t), & x=y=z, \\ \mathcal{D}\left(\frac{t}{G(x, y, z)}\right), & \text { otherwise }\end{cases}
$$

where $H(t)$ and $\mathcal{D}(t)$ are distribution functions as follows:

$$
H(t)=\left\{\begin{array}{ll}
0, & t \leq 0, \\
t, & t>0 .
\end{array}, \quad \mathcal{D}= \begin{cases}0, & t \leq 0 \\
1-e^{-t}, & t>0\end{cases}\right.
$$


Now, consider the following sequence in $\mathbb{R}$ :

$$
x_{n}= \begin{cases}n, & n \text { is square } \\ 1, & \text { otherwise }\end{cases}
$$

It is clear that $\left\{x_{n}\right\}$ statistically converges to 1 but it is not convergent normally.

Definition 3.10 A set $A=\left\{n_{k}: k \in \mathbb{N}\right\}$ is said to be statistically dense in $\mathbb{N}$ if the set

$$
A(n)=\left\{\left(i_{1}, i_{2}, \ldots, i_{l}\right) \in A^{l}, i_{1}, i_{2}, \ldots, i_{l} \leq n\right\}
$$

has asymptotic density 1 , i.e.,

$$
\delta_{l}(A)=\lim _{n \rightarrow \infty} \frac{l !|A(n)|}{n^{l}}=1 .
$$

Theorem 3.11 Let $\left\{x_{n}\right\}$ be a sequence in the PGM-space $(X, F, T)$. Then the following are equivalent:

(i) $\left\{x_{n}\right\}$ statistically converges to a point $x \in X$.

(ii) There is a sequence $\left\{y_{n}\right\}$ in $X$ such that $x_{n}=y_{n}$ for almost all $n$, and $\left\{y_{n}\right\}$ converges to $x$.

(iii) There is a statistically dense subsequence $\left\{x_{n_{k}}\right\}$ of $\left\{x_{n}\right\}$ such that $\left\{x_{n_{k}}\right\}$ is convergent.

(iv) There is a statistically dense subsequence $\left\{x_{n_{k}}\right\}$ of $\left\{x_{n}\right\}$ such that $\left\{x_{n_{k}}\right\}$ is statistically convergent.

Proof $(i \Longrightarrow i i)$ Let $\left\{x_{n}\right\}$ be a sequence that converges to $x$, so

$$
\begin{aligned}
& \delta_{l}\left(\left\{\left(i_{1}, i_{2}, \ldots, i_{l}\right) \in \mathbb{N}^{l}: F_{\left(x_{i_{1}}, x_{i_{2}}, \ldots, x_{i}, x\right)}(\epsilon)>1-\delta\right\}\right) \\
& \quad=\lim _{n \rightarrow \infty} \frac{l !}{n^{l}}\left|\left\{\left(i_{1}, i_{2}, \ldots, i_{l}\right) \in \mathbb{N}^{l} ; i_{1}, i_{2}, \ldots, i_{l} \leq n, F_{\left(x_{i_{1}}, x_{i_{2}}, \ldots, x_{i_{l}}, x\right)}(\epsilon)>1-\delta\right\}\right|=1 .
\end{aligned}
$$

For each $k \in \mathbb{N}$, we can choose an increasing sequence $\left\{n_{k}\right\}$ such that, for every $n>n_{k}$,

$$
\frac{l !}{n^{l}}\left|\left\{\left(i_{1}, i_{2}, \ldots, i_{l}\right) \in \mathbb{N}^{l} ; i_{1}, i_{2}, \ldots, i_{l} \leq n, F_{\left(x_{i_{1}}, x_{i_{2}}, \ldots, x_{i_{l}}, x\right)}(\epsilon)>1-\frac{1}{2^{k}}\right\}\right|>1-\frac{1}{2^{k}} .
$$

Define the sequence $\left\{y_{n}\right\}$ as follows:

$$
y_{m}= \begin{cases}x_{m}, & 1 \leq m \leq n_{1} \\ x_{m}, & n_{k}<m \leq n_{k+1}, i_{1}, i_{2}, \ldots, i_{l-1} \leq n_{k+1}, F_{\left(x_{i_{1}}, x_{i_{2}}, \ldots, x_{i_{l-1}}, x_{m}, x\right)}(\epsilon)>1-\frac{1}{2^{k}}, \\ x, & \text { otherwise. }\end{cases}
$$

Choose $k \in \mathbb{N}$ such that $\frac{1}{2^{k}}<\delta$. It is clear that $\left\{y_{m}\right\}$ converges to $x$. Fix $n \in \mathbb{N}$, for $n_{k}<n \leq$ $n_{k+1}$, we have

$$
\begin{aligned}
& \left\{\left(i_{1}, i_{2}, \ldots, i_{l}\right) \in \mathbb{N}^{l} ; i_{1}, i_{2}, \ldots, i_{l} \leq n ; x_{i_{j}} \neq y_{i_{j}}\right\} \\
& \subseteq \\
& \quad\left\{\left(i_{1}, i_{2}, \ldots, i_{l}\right) \in \mathbb{N}^{l} ; i_{1}, i_{2}, \ldots, i_{l} \leq n\right\} \\
& \quad-\left\{\left(i_{1}, i_{2}, \ldots, i_{l}\right) \in \mathbb{N}^{l} ; i_{1}, i_{2}, \ldots, i_{l} \leq n_{k}, F_{\left(x_{i_{1}}, x_{i_{2}}, \ldots, x_{i_{l}}, x\right)}(\epsilon)>1-\frac{1}{2^{k}}\right\} .
\end{aligned}
$$


Hence,

$$
\begin{aligned}
& \lim _{n \rightarrow \infty} \frac{l !}{n^{l}}\left|\left\{\left(i_{1}, i_{2}, \ldots, i_{l}\right) \in \mathbb{N}^{l} ; i_{1}, i_{2}, \ldots, i_{l} \leq n ; x_{i_{j}} \neq y_{i_{j}}\right\}\right| \\
& \quad \leq 1-\lim _{n \rightarrow \infty} \frac{l !}{n^{l}}\left|\left\{\left(i_{1}, i_{2}, \ldots, i_{l}\right) \in \mathbb{N}^{l} ; i_{1}, i_{2}, \ldots, i_{l} \leq n_{k}, F_{\left(x_{i_{1}}, x_{i_{2}}, \ldots, x_{i_{l}}, x\right)}(\epsilon)>1-\frac{1}{2^{k}}\right\}\right| \\
& \quad<\frac{1}{2^{k}}<\delta
\end{aligned}
$$

so

$$
\begin{aligned}
& \delta_{l}\left(\left\{\left(i_{1}, i_{2}, \ldots, i_{l}\right) \in \mathbb{N}^{l} ; i_{1}, i_{2}, \ldots, i_{l} \leq n ; x_{i_{j}} \neq y_{i_{j}}\right\}\right) \\
& \quad=\lim _{n \rightarrow \infty} \frac{l !}{n^{l}}\left|\left\{\left(i_{1}, i_{2}, \ldots, i_{l}\right) \in \mathbb{N}^{l} ; i_{1}, i_{2}, \ldots, i_{l} \leq n ; x_{i_{j}} \neq y_{i_{j}}\right\}\right|=0 .
\end{aligned}
$$

(ii $\Longrightarrow$ iii) Let $\left\{y_{n}\right\}$ be a convergent sequence in $X$ and $A=\left\{n \in \mathbb{N}: y_{n} \neq x_{n}\right\}$. We have $\delta_{l}(A)=1$, so the sequence $\left\{y_{n}\right\}$ is a statistical dense subsequence of $\left\{x_{n}\right\}$ that is convergent.

$(i i i \Longrightarrow i v)$ It is obvious from Theorem 3.8.

$(i v \Longrightarrow i)$ Let $\left\{x_{n_{k}}\right\}$ be a statistically dense subsequence of $\left\{x_{n}\right\}$ that is statistically convergent to a point $x \in X$. Set $A=\left\{n_{k}: k \in \mathbb{N}\right\}$, so $\delta_{l}(A)=1$. For $\epsilon>$ and $0<\delta<1$,

$$
\begin{aligned}
& \left\{\left(i_{1}, i_{2}, \ldots, i_{l}\right) \in \mathbb{N}^{l} ; i_{1}, i_{2}, \ldots, i_{l} \leq n, F_{\left(x_{i_{1}}, x_{i_{2}}, \ldots, x_{i_{l}}, x\right)}(\epsilon)>1-\delta\right\} \\
& \quad \supseteq\left\{\left(i_{1}, i_{2}, \ldots, i_{l}\right) \in \mathbb{A}^{l} ; i_{1}, i_{2}, \ldots, i_{l} \leq n, F_{\left(x_{i_{1}}, x_{i_{2}}, \ldots, x_{l}, x\right)}(\epsilon)>1-\delta\right\} .
\end{aligned}
$$

Hence,

$$
\begin{aligned}
& \lim _{n \rightarrow \infty} \frac{l !}{n^{l}}\left|\left\{\left(i_{1}, i_{2}, \ldots, i_{l}\right) \in \mathbb{N}^{l} ; i_{1}, i_{2}, \ldots, i_{l} \leq n, F_{\left(x_{i_{1}}, x_{i_{2}}, \ldots, x_{i_{l}}, x\right)}(\epsilon)>1-\delta\right\}\right| \\
& \quad \geq \lim _{n \rightarrow \infty} \frac{l !}{n^{l}}\left|\left\{\left(i_{1}, i_{2}, \ldots, i_{l}\right) \in \mathbb{A}^{l} ; i_{1}, i_{2}, \ldots, i_{l} \leq n, F_{\left(x_{i_{1}}, x_{i_{2}}, \ldots, x_{i_{l}}, x\right)}(\epsilon)>1-\delta\right\}\right|=1 .
\end{aligned}
$$

So,

$$
\delta_{l}\left(\left\{\left(i_{1}, i_{2}, \ldots, i_{l}\right) \in \mathbb{N}^{l} ; i_{1}, i_{2}, \ldots, i_{l} \leq n, F_{\left(x_{i_{1}}, x_{i_{2}}, \ldots, x_{i_{l}}, x\right)}(\epsilon)>1-\delta\right\}\right)=1 .
$$

Therefore $\left\{x_{n}\right\}$ statistically converges to $x$.

The following corollary is a direct consequence of the above theorem.

Corollary 3.12 Every statistically convergent sequence in a PGM-space has a convergent subsequence.

Theorem 3.13 Every statistically convergent sequence in a PGM-space is statistically Cauchy.

Proof Suppose that $\left\{x_{n}\right\}$ is a sequence that statistically converges to a point $x$. Let $\epsilon>0$ and $0<\delta<1$. Since $T$ is continuous, there are $0<\delta_{1}<1$ and $0<\delta_{2}<1$ such that $T(1-$ 
$\left.\delta_{1}, 1-\delta_{2}\right)>1-\delta$. On the other hand, there exists $i_{\epsilon}$ such that

$$
F_{\left(x_{\epsilon}, x, x, \ldots, x\right)}\left(\frac{\epsilon}{2}\right)>1-\delta_{1}
$$

Since

$$
F_{\left(x_{i_{1}}, x_{i_{2}}, \ldots, x_{i}, x\right)}(\epsilon) \geq T\left(F_{\left(x_{i_{\epsilon}}, x, \ldots, x\right)}\left(\frac{\epsilon}{2}\right), F_{\left(x_{i_{1}}, x_{i_{2}}, \ldots, x_{i_{l}}, x_{i_{\epsilon}}\right)}\left(\frac{\epsilon}{2}\right)\right)
$$

So

$$
\begin{aligned}
& \left\{\left(i_{1}, i_{2}, \ldots, i_{l}\right) \in \mathbb{N}^{l} ; i_{1}, i_{2}, \ldots, i_{l} \leq n, F_{\left(x_{i_{1}}, x_{i_{2}}, \ldots, x_{i_{l}}, x_{i_{\epsilon}}\right)}\left(\frac{\epsilon}{2}\right)>1-\delta_{2}\right\} \\
& \subseteq\left\{\left(i_{1}, i_{2}, \ldots, i_{l}\right) \in \mathbb{N}^{l} ; i_{1}, i_{2}, \ldots, i_{l} \leq n, F_{\left(x_{i_{1}}, x_{i_{2}}, \ldots, x_{i}, x\right)}(\epsilon)>1-\delta\right\} .
\end{aligned}
$$

Hence

$$
\begin{aligned}
& \lim _{n \rightarrow \infty} \frac{l !}{n^{l}}\left|\left\{\left(i_{1}, i_{2}, \ldots, i_{l}\right) \in \mathbb{N}^{l} ; i_{1}, i_{2}, \ldots, i_{l} \leq n, F_{\left(x_{i_{1}}, x_{i_{2}}, \ldots, x_{i_{l}}, x_{i_{\epsilon}}\right)}\left(\frac{\epsilon}{2}\right)>1-\delta_{2}\right\}\right| \\
& \leq \lim _{n \rightarrow \infty} \frac{l !}{n^{l}}\left|\left\{\left(i_{1}, i_{2}, \ldots, i_{l}\right) \in \mathbb{N}^{l} ; i_{1}, i_{2}, \ldots, i_{l} \leq n, F_{\left(x_{i_{1}}, x_{i_{2}}, \ldots, x_{i}, x\right)}(\epsilon)>1-\delta\right\}\right| .
\end{aligned}
$$

Since $\left\{x_{n}\right\}$ is statistically convergent, so the right-hand side of the previous inequality is zero. Therefore it shows that the sequence $\left\{x_{n}\right\}$ is statistically Cauchy.

Definition 3.14 Let $(X, F, T)$ be a $P G M$-space. If every statistically Cauchy sequence is statistically convergent, then $(X, F, T)$ is said to be statistically complete.

Corollary 3.15 Every statistically complete PGM-space is complete.

Proof Let $(X, F, T)$ be a statistically complete $P G M$-space. Suppose that $\left\{x_{n}\right\}$ is a Cauchy sequence in $(X, F, T)$, so it is a statistically Cauchy sequence. Since $X$ is statistically complete, so $\left\{x_{n}\right\}$ is statistically convergent. By Corollary 3.12 , there is a subsequence $\left\{x_{n_{k}}\right\}$ of $\left\{x_{n}\right\}$ that converges to a point $x \in X$. By the continuity of $T$, for $0<\delta<1$, there exist $0<\delta_{1}, \delta_{2}, \delta_{3}, \delta_{4}<1$ such that

$$
\left\{\begin{array}{l}
T\left(1-\delta_{1}, 1-\delta_{2}\right)>1-\delta \\
T\left(1-\delta_{3}, 1-\delta_{4}\right)>1-\delta_{1}
\end{array}\right.
$$

Let $\delta_{5}:=\max \left\{\delta_{2}, \delta_{3}\right\}$, then we have

$$
T\left(T\left(1-\delta_{5}, 1-\delta_{4}\right), 1-\delta_{5}\right)>1-\delta
$$

For $\epsilon>0$, since $\left\{x_{n}\right\}$ is Cauchy, then there exist $N_{1} \in \mathbb{N}$ and $x_{i_{\epsilon}} \in\left\{x_{n}\right\}$ such that, for all $i_{1}, i_{2}, \ldots, i_{l} \geq N_{1}$,

$$
F_{\left(x_{i_{1}}, x_{i_{2}}, \ldots, x_{i_{l}}, x_{i_{\epsilon}}\right)}\left(\frac{\epsilon}{4}\right)>1-\delta_{5}
$$


and since $x_{n_{k}} \longrightarrow x$, there exists $N_{2} \geq N_{1}$ such that, for $i_{n_{1}}, i_{n_{2}}, \ldots, i_{n_{l}} \geq N_{2}$,

$$
F_{\left(x_{i_{1}}, x_{i_{2}}, \ldots, x_{i_{l}}, x\right)}\left(\frac{\epsilon}{4}\right)>1-\delta_{5}
$$

For $i_{1}, i_{2}, \ldots, i_{l}, i_{n_{1}}, i_{n_{2}}, \ldots, i_{n_{l}} \geq N_{2}$, we have

$$
\begin{aligned}
& F_{\left(x_{i_{1}}, x_{i_{2}}, \ldots, x_{i}, x\right)}(\epsilon) \\
& \geq T\left(F_{\left(x_{i \epsilon}, x, x, \ldots, x\right)}\left(\frac{\epsilon}{2}\right), F_{\left(x_{i_{1}}, x_{i_{2}}, \ldots, x_{i}, x_{i_{\epsilon}}\right)}\left(\frac{\epsilon}{2}\right)\right) \\
& \geq T\left(T\left(F_{\left(x_{i_{\epsilon}}, x_{i_{1}}, x_{i_{1}}, \ldots, x_{i_{1}}\right)}\left(\frac{\epsilon}{4}\right), F_{\left(x_{i_{1}}, x, x, \ldots, x\right)}\left(\frac{\epsilon}{4}\right)\right), F_{\left(x_{i_{1}}, x_{i_{2}}, \ldots, x_{i_{l}}, x_{i_{\epsilon}}\right)}\left(\frac{\epsilon}{2}\right)\right) \\
& \geq T\left(T\left(F_{\left(x_{i_{\epsilon}}, x_{i_{1}}, x_{i_{2}}, \ldots, x_{i_{l}}\right)}\left(\frac{\epsilon}{4}\right), F_{\left(x_{i_{1}}, x_{i_{1}}, x_{i_{2}}, \ldots, x_{i_{l}}\right)}\left(\frac{\epsilon}{4}\right)\right), F_{\left(x_{i_{1}}, x_{i_{2}}, \ldots, x_{i}, x_{i_{\epsilon}}\right)}\left(\frac{\epsilon}{4}\right)\right) \\
& >T\left(T\left(1-\delta_{5}, 1-\delta_{4}\right), 1-\delta_{5}\right) \\
& >1-\delta \text {. }
\end{aligned}
$$

The third inequality arises from part (ii) of Definition 3.3 and the nondecreasing property of $F$. So, $\left\{x_{n}\right\}$ is convergent and therefore $(X, F, T)$ is complete.

\section{Acknowledgements}

The author received no financial support for the research, authorship, or publication of this article.

Funding

Not applicable.

\section{Availability of data and materials}

Not applicable.

\section{Competing interests}

The author declares that they have no competing interests.

\section{Authors' contributions}

The author read and approved the final manuscript.

\section{Publisher's Note}

Springer Nature remains neutral with regard to jurisdictional claims in published maps and institutional affiliations.

Received: 23 March 2021 Accepted: 14 July 2021 Published online: 30 July 2021

\section{References}

1. Abazari, R.: Statistical convergence in g-metric spaces. arxiv:2103.05527v1 (2021)

2. Alotaibi, A., Mursaleen, M.: Statistical convergence in random paranormed space. J. Comput. Anal. Appl. 17(2) 297-304 (2014)

3. Choi, H. Kim, S., Yang, S.: Structure for g-metric spaces and related fixed point theorem. arxiv:1804.03651v1 (2018)

4. Connor, J., Kline, J.: On statistical limit points and the consistency of statistical convergence. J. Math. Anal. Appl. 197, 392-399 (1996)

5. Duman, O., Khan, M.K., Orhan, C.: A-Statistical convergence of approximating operators. Math. Inequal. Appl. 6, 689-699 (2003)

6. Erdös, P., Tenenbaum, G.: Sur les densities de certeines suites d'entiers. Proc. Lond. Math. Soc. 3(59), 417-438 (1989)

7. Fast, H.: Sur la convergence statistique. Colloq. Math. 2, 241-244 (1951)

8. Fridy, J.A.: On statistical convergence. Analysis 5, 301-313 (1985)

9. Fridy, J.A., Miller, H.I.: A matrix characterization of statistical convergence. Analysis 11, $59-66$ (1991)

10. Jena, B.B., Paikray, S.K., Dutta, H.: On various new concepts of statistical convergence for sequences of random variables via deferred Cesaro mean. J. Math. Anal. Appl. 487, 123950 (2020)

11. Maddox, I.J.: Statistical convergence in a locally convex space. Math. Proc. Camb. Philos. Soc. 104, 141-145 (1988)

12. Menger, K.: Statistical metrics. Proc. Natl. Acad. Sci. USA 28, 535-537 (1942) 
13. Mursaleen, M., Danish Lohani, Q.M.: Statistical limit superior and limit inferior in probabilistic normed spaces. Filomat 25(3), 55-67 (2011)

14. Mursaleen, M., Mohiuddine, S.A.: On ideal convergence of double sequences in probabilistic normed spaces. Math. Rep. 12(62), 359-371 (2010)

15. Mursaleen, M., Mohiuddine, S.A.: On ideal convergence in probabilistic normed spaces. Math. Slovaca 62(1), 49-62 (2012)

16. Mustafa, Z., Sims, B.: A new approach to generalized metric spaces. J. Nonlinear Convex Anal. 7(2), 289-297 (2006)

17. Pehlivan, S., Güncan, A., Mamedov, A.: On statistical convergent sequences of real numbers. Math. Slovaca 30, 139-150 (1980)

18. Raj, K., Anand, R.: On statistical convergence in generalized lacunary sequence spaces. Bul. Acad. Ştiinţe Repub. Mold. Mat. 2, 17-29 (2018)

19. Raj, K., Jamwal, S.: Applications of statistical convergence to n-normed spaces. Adv. Pure Appl. Math. 7(3), 197-204 (2016)

20. Raj, K., Pandoh, S., Devi, M.: Orlicz b-metric spaces of infinite matrix and their Kothe-Toeplitz duals. J. Interdiscip. Math. 21(7-8), 1587-1595 (2018)

21. Salat, T.: Statistical cluster points of sequences in finite dimensional spaces. Czechoslov. Math. J. 54, 95-102 (2004)

22. Schoenberg, I.J.: The integrability of certain functions and related summability methods. Am. Math. Mon. 66, 361-375 (1959)

23. Schweizer, B.: Probabilistic Metric Space. Elsevier, New York (1983)

24. Sencimen, C., Pehlivan, S.: Strong statistical convergence in probabilistic metric spaces. Stoch. Anal. Appl. 26, 651-664 (2008)

25. Steinhaus, H.: Sur la convergence ordinaire et la convergence asymptotique. Colloq. Math. 2, 73-74 (1951)

26. Zhou, C., Wang, S., Ciric, L., Alsulami, S.: Generalized probabilistic metric spaces and fixed point theorems. Fixed Point Theory Appl. 2014, 91 (2014)

27. Zygmund, A.: Trigonometric Series. Cambridge University Press, Cambridge (1979)

\section{Submit your manuscript to a SpringerOpen ${ }^{\circ}$ journal and benefit from:}

- Convenient online submission

- Rigorous peer review

- Open access: articles freely available online

- High visibility within the field

- Retaining the copyright to your article

Submit your next manuscript at $\boldsymbol{\nabla}$ springeropen.com 